OPEN ACCESS

Edited by:

Kürşad Turgay,

Max-Planck-Gesellschaft (MPG),

Germany

Reviewed by:

Christiane Wolz,

University of Tübingen, Germany

Walid A. Houry,

University of Toronto, Canada

Mark Brönstrup,

Helmholtz Association of German

Research Centers (HZ), Germany

${ }^{*}$ Correspondence:

Dorte Frees

df@sund.ku.dk

Specialty section:

This article was submitted to

Protein Folding, Misfolding,

and Degradation,

a section of the journal

Frontiers in Molecular Biosciences

Received: 06 April 2021

Accepted: 14 May 2021

Published: 04 June 2021

Citation:

Bæk KT, Jensen C, Farha MA Nielsen TK, Paknejadi E, Mebus VH, Vestergaard $M$, Brown ED and Frees $D$ (2021) A Staphylococcus aureus clpX Mutant Used as a Unique Screening

Tool to Identify Cell Wall Synthesis

Inhibitors that Reverse $\beta$-Lactam

Resistance in MRSA.

Front. Mol. Biosci. 8:691569.

doi: 10.3389/fmolb.2021.691569

\section{A Staphylococcus aureus clpX Mutant Used as a Unique Screening Tool to Identify Cell Wall Synthesis Inhibitors that Reverse $\beta$-Lactam Resistance in MRSA}

\author{
Kristoffer T. Bæk ${ }^{1}$, Camilla Jensen ${ }^{1}$, Maya A. Farha ${ }^{2}$, Tobias K. Nielsen ${ }^{1}$, Ervin Paknejadi ${ }^{1}$, \\ Viktor H. Mebus ${ }^{1}$, Martin Vestergaard ${ }^{1}$, Eric D. Brown ${ }^{2}$ and Dorte Frees ${ }^{1 *}$ \\ ${ }^{1}$ Department of Veterinary and Animal Sciences, Faculty of Health and Medical Sciences, University of Copenhagen, \\ Copenhagen, Denmark, ${ }^{2}$ Department of Biochemistry and Biomedical Sciences, Michael G. DeGroote Institute for Infectious \\ Disease Research, McMaster University, Hamilton, ON, Canada
}

Staphylococcus aureus is a leading cause of bacterial infections world-wide. Staphylococcal infections are preferentially treated with $\beta$-lactam antibiotics, however, methicillin-resistant $S$. aureus (MRSA) strains have acquired resistance to this superior class of antibiotics. We have developed a growth-based, high-throughput screening approach that directly identifies cell wall synthesis inhibitors capable of reversing $\beta$-lactam resistance in MRSA. The screen is based on the finding that $S$. aureus mutants lacking the ClpX chaperone grow very poorly at $30^{\circ} \mathrm{C}$ unless specific steps in teichoic acid synthesis or penicillin binding protein (PBP) activity are inhibited. This property allowed us to exploit the $S$. aureus clpX mutant as a unique screening tool to rapidly identify biologically active compounds that target cell wall synthesis. We tested a library of $\sim 50,000$ small chemical compounds and searched for compounds that inhibited growth of the wild type while stimulating growth of the clpX mutant. Fifty-eight compounds met these screening criteria, and preliminary tests of 10 compounds identified seven compounds that reverse $\beta$-lactam resistance of MRSA as expected for inhibitors of teichoic acid synthesis. The hit compounds are therefore promising candidates for further development as novel combination agents to restore $\beta$-lactam efficacy against MRSA.

Keywords: ClpX, Staphylococcus aureus, cell wall synthesis, teichoic acid inhibitors, high-throughput screen, pathway-directed drug discovery, $\beta$-lactam antibiotics

\section{INTRODUCTION}

There is an unmet need for novel antibiotics to tackle the challenges associated with the world-wide dissemination of antibiotic resistant bacterial pathogens such as methicillin resistant Staphylococcus aureus (MRSA) (Tacconelli et al., 2018; Vestergaard et al., 2019). A common approach for identification of compounds with antibacterial activity is to screen large libraries of small molecules for compounds that inhibit bacterial growth. Whole cell screens based on growth inhibition are easily carried out in a high-throughput format, however, a major disadvantage of whole cell screens is that target identification is often challenging and time-consuming (French et al., 2017). In addition, whole cell screens for growth inhibition typically generate large numbers of active 
compounds, many of which have non-specific activities (Silver, 2011). Therefore, including a counter-screen that facilitates exclusion of non-specific inhibitors and allows identification of compounds targeting specific pathways early in the screening workflow can speed up the screening process tremendously (French et al., 2017; Buss et al., 2018).

In this report, we describe the development of a counterscreen that enables identification of compounds targeting cell wall synthesis in the major human pathogenic bacterium, S. aureus. The screen is based on growth (measured as change in absorbance) of an $S$. aureus mutant that lacks the ClpX chaperone, and the screen is therefore well suited for a highthroughput approach.

In all living cells, molecular chaperones are essential for facilitating folding and unfolding of proteins (Olivares et al., 2016). ClpX is a highly conserved ATP-dependent unfoldase that can associate with ClpP proteolytic subunits to form the ClpXP protease (Baker and Sauer, 2012). In S. aureus, deletion of the clpX gene confers a cold-sensitive phenotype characterized by severely reduced final yield at $30^{\circ} \mathrm{C}$ (Frees et al., 2003; Bæk et al., 2016). Remarkably, the poor growth of $S$. aureus clpX mutants can be rescued by inhibiting specific steps in the biosynthesis pathway of peptidoglycan or teichoic acids, the two major components of the Gram-positive cell wall (Bæk et al., 2016; Jensen et al., 2019). For example, $\beta$-lactam antibiotics, which inhibit cross-linking of peptidoglycan by binding irreversibly to the penicillin-binding proteins (PBPs), increase the growth yield of the $S$. aureus $c l p X$ mutant up to six times when added at sublethal concentrations (Jensen et al., 2019). Similarly, the antibiotics tunicamycin and tarocin A1 which both inhibit the TarO enzyme in the wall teichoic acid (WTA) biosynthesis pathway rescue growth of the clpX mutant, whereas other classes of antibiotics with different cellular targets, or inhibiting other steps in WTA or peptidoglycan synthesis have no effect (Jensen et al., 2019). Moreover, growth of S. aureus clpX mutants can be rescued genetically by inactivating the lipoteichoic acid synthase (LtaS) that catalyzes the last step in lipoteichoic acid (LTA) biosynthesis, as revealed by the characterization of spontaneous suppressor mutations acquired by $S$. aureus clpX strains (Bæk et al., 2016). LTA biosynthesis, similarly to WTA synthesis, is conditionally essential and an attractive target for novel antibiotics (Richter et al., 2013; Sewell and Brown, 2014; Coe et al., 2019).

Based on these findings we reasoned that an S. aureus clpX mutant could work as a screening tool to identify antimicrobial compounds targeting cell wall synthesis of $S$. aureus. Compounds that rescue growth of the $S$. aureus $c l p X$ mutant are predicted to inhibit crosslinking of peptidoglycan, or to inhibit specific steps in LTA synthesis, or WTA synthesis. Importantly, a number of elegant studies demonstrated that MRSA strains are sensitized to $\beta$-lactams if WTA or LTA biosynthesis is inhibited (Campbell et al., 2011; Farha et al., 2013, Roemer et al., 2013, Lee et al., 2016). Therefore, screened out compounds with a target in teichoic acid biosynthesis would have potential to be used in combination with $\beta$-lactams for treatment of MRSA-infections.

To test this hypothesis we set up the screening platform as follows. First, we identified compounds that inhibit growth of
S. aureus from a library of 50,000 small chemical compounds. Second, the subset of $S$. aureus active compounds was deployed in the counter-screen to identify compounds that improve the growth yield of the $S$. aureus clpX mutant. From the initial 50,000 compounds, we identified 828 compounds with antimicrobial activity against $S$. aureus, and 58 of these enhanced growth of the clpX mutant indicating that they target cell wall synthesis. Finally, a subset of ten compounds was further tested, and seven out of seven hit compounds sensitized an MRSA strain to $\beta$-lactam antibiotics, demonstrating the power of the screen at identifying compounds that can restore antibiotic sensitivity in MRSA.

\section{METHODS}

\section{Bacterial Strains and Growth Conditions}

$S$. aureus strains used in this study were the methicillin sensitive clinical isolate, SA564 (Somerville et al., 2002), SA564 clpX (Jelsbak et al., 2010) and the MRSA strains USA300 JE2 (Fey et al., 2013), and COL (Dyke et al., 1966). S. aureus strains were cultured in tryptic soy broth [TSB (Oxoid)] at 37 or $30^{\circ} \mathrm{C}$ with eration, or on TSB medium solidified with $1.5 \%$ (wt/vol) agar (TSA). When inoculating the $c l p X$ deletion strain, care was taken to avoid visibly larger colonies containing potential suppressor mutants (Brk et al., 2016).

\section{Primary Screen}

Screening for S. aureus growth inhibition was performed in 384well microtiter plates (catalog no. 3701, Corning) in duplicate using a stand-alone Biomek FXP integrated liquid handler (Beckman Coulter). The screening library consisted of 50,000 small drug-like chemical compounds from the Maybridge screening collection (ThermoFisher). The evening before screening, a single colony of wild type $S$. aureus SA564 was inoculated into $5 \mathrm{ml}$ of TSB and grown overnight at $37^{\circ} \mathrm{C}$. On the day of screening, the overnight culture was diluted 1:200 in TSB and grown to mid-exponential phase $\left(\mathrm{OD}_{600}\right.$ of $\left.\sim 0.5\right)$. Cells were then diluted into fresh TSB to a final $\mathrm{OD}_{600}$ of 0.001 . The Biomek FXP liquid handler was used to dispense in duplicate $20 \mu \mathrm{l}$ of TSB followed by $0.4 \mu \mathrm{l}$ of each compound of the 50,000 smallmolecule library ( $1 \mathrm{mM}$ stock dissolved in 100\% DMSO) into each well. The liquid handler was then subsequently used to dispense $20 \mu \mathrm{l}$ of culture ( $S$. aureus $\mathrm{SA} 564 \mathrm{OD}_{600} 0.001$ ), giving a final screening concentration of $10 \mu \mathrm{M}$. $1 \%$ DMSO, and $1 \%$ $\mathrm{DMSO}+2.5 \mathrm{mg} \mathrm{L}^{-1}$ erythromycin were used as high and low controls, respectively. Plates were incubated at $37^{\circ} \mathrm{C}$ in a Cytomat stationary incubator (ThermoFisher) for $8 \mathrm{~h}$. These conditions resulted in a $Z^{\prime}$ value of 0.8 (Supplementary Figure S1A). After incubation, absorbance was read at $600 \mathrm{~nm}$ using an EnVision plate reader (PerkinElmer). Data were normalized to take into account both plate and well positional effects using a method previously described (Mangat et al., 2014). A statistical cutoff of 3 standard deviations below the mean of the data set was established to select active compounds.

To confirm the activity of the 993 selected $S$. aureus active compounds, a half-log serial dilution series (50 nM-5 mM) of 
TABLE 1 | Hit-compounds listed according to their ability to increase the growth yield of SA564 c/pX.

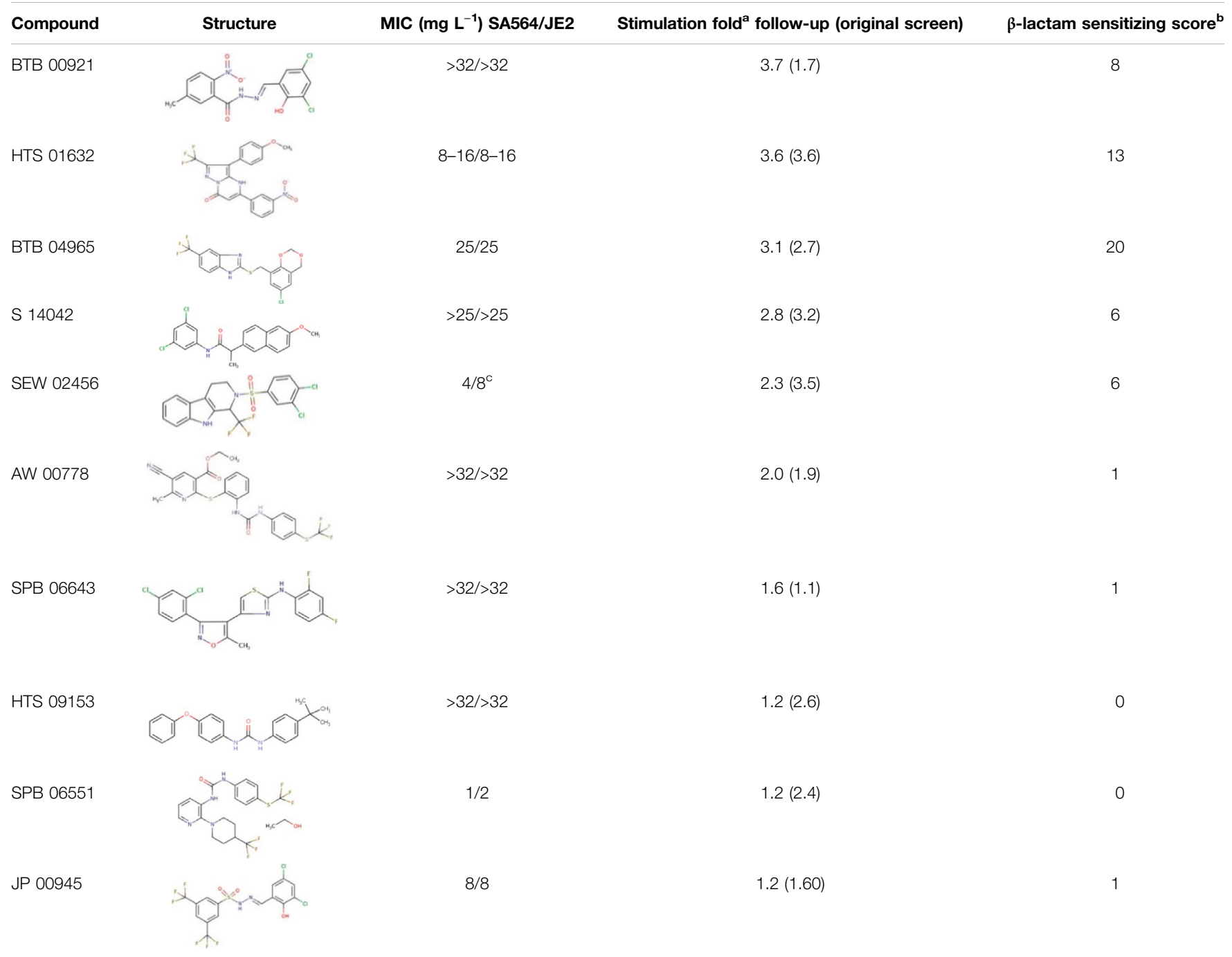

${ }^{a}$ The fold increase in final yield $\left(O D_{600}\right)$ of a S. aureus clpX mutant obtained in the follow-up assay, and in the original screen (value in parenthesis).

${ }^{b}$ The sensitizing score was calculated based on the summarized values given in Figure 4 (see legend to this figure for details).

${ }^{c}$ Growth completely inhibited at 4-8 $\mathrm{mg} \mathrm{L}^{-1}$ SEW 02456 but limited growth observed at higher compound concentrations due to precipitation of compound.

each compound was prepared in DMSO. $1 \mu \mathrm{l}$ of each dilution was dispensed in duplicate into dry wells on 96-well microtiter plates (catalog no. 3370, Corning) using a Biomek FX liquid handler, and a Freedom EVO liquid handler (Tecan) was then used to dispense $99 \mu \mathrm{l}$ of culture (S. aureus SA564 $\mathrm{OD}_{600}=0.001$ ) prepared as described above, giving a final concentration range of $0.5 \mathrm{nM}-50 \mu \mathrm{M}$. Eight $1 \%$ DMSO wells were included on each plate as no-compound controls. Plates were then incubated at $37^{\circ} \mathrm{C}$ with shaking (600 rpm) for $7 \mathrm{~h}$. After incubation, absorbance was read at $600 \mathrm{~nm}$ using an Infinite M1000 Pro plate reader (Tecan). To take into account plate positional effects, data for each plate were normalized to the mean of the DMSO wells excluding the two lowest and two highest values. The doseresponse relationship of 828 of the compounds resulted in a typical sigmoidal semi-logarithmic curve associated with growth inhibition. 165 compounds failed to inhibit growth in this assay and were discarded from the downstream analyses.

\section{Counter Screen: Growth Stimulation of S. aureus clpX Mutant}

The evening before screening, a single small colony of $S$. aureus SA564 clpX was picked from a plate incubated at $37^{\circ} \mathrm{C}$ and inoculated into $1 \mathrm{ml}$ TSB that was then incubated overnight at $37^{\circ} \mathrm{C}$. On the day of screening, the overnight culture was diluted 1: 200 in TSB and grown to mid-exponential phase $\left(\mathrm{OD}_{600}\right.$ of $0.3-0.6)$ at $37^{\circ} \mathrm{C}$. Cells were diluted into fresh TSB to a final $\mathrm{OD}_{600}$ of 0.1 , and then diluted 1:10,000 into $400 \mathrm{ml} \mathrm{TSB}$. A half$\log$ serial dilution series $(50 \mathrm{nM}-5 \mathrm{mM})$ of each $S$. aureus active compound was prepared in DMSO, and $1 \mu$ of each dilution was dispensed in duplicate into dry wells on 96-well microtiter plates (catalog no. 3370, Corning) using a Biomek FX liquid handler. A Freedom EVO liquid handler (Tecan) was then used to dispense $99 \mu \mathrm{l}$ of the prepared $S$. aureus $c l p X$ culture, giving a final concentration range of $0.5 \mathrm{nM}-50 \mu \mathrm{M}$. Eight $1 \%$ DMSO wells 
were included on each plate as no-compound controls. Plates were then incubated at $30^{\circ} \mathrm{C}$ with shaking $(600 \mathrm{rpm})$ for $24 \mathrm{~h}$. After incubation, absorbance was read at $600 \mathrm{~nm}$ using an Infinite M1000 Pro plate reader (Tecan). To take into account plate positional effects, data for each plate were normalized to the mean of the DMSO wells excluding the two lowest and two highest values. For each compound and each dose, the lowest normalized OD value of replicates 1 and 2 was used to determine an increase in final growth yield, and the highest of these values for each compound across all doses was used as the screen read-out. A compound was classified as active if this value was 1.5 or higher.

\section{Disk Diffusion Assay}

The MRSA strain COL was inoculated on TSA plates and incubated at $37^{\circ} \mathrm{C}$ overnight. The next day, bacterial colonies were suspended in $0.9 \% \mathrm{NaCl}$, adjusted to $0.5 \mathrm{McF}$ arland (Sensititre $^{\circledR}$ nephelometer and the Sensititre ${ }^{\circledR}$ McFarland Standard), and streaked on TSA plates with or without the following compounds: BTB $00921 \quad\left(4 \mathrm{mg} \mathrm{L}^{-1}\right)$, HTS 01632 $\left(6 \mathrm{mg} \mathrm{L}^{-1}\right)$, BTB $04965\left(3 \mathrm{mg} \mathrm{L}^{-1}\right)$, S $14042\left(3 \mathrm{mg} \mathrm{L}^{-1}\right)$, SEW $02456\left(6 \mathrm{mg} \mathrm{L}^{-1}\right)$, AW $00778\left(6 \mathrm{mg} \mathrm{L}^{-1}\right)$, SPB $06643\left(5 \mathrm{mg} \mathrm{L}^{-1}\right)$, HTS $09153\left(2 \mathrm{mg} \mathrm{L}^{-1}\right)$, SPB $06551\left(0.2 \mathrm{mg} \mathrm{L}^{-1}\right)$, and JP 00945 $\left(2 \mathrm{mg} \mathrm{L}^{-1}\right)$. The plates were allowed to dry prior to the addition of antibiotic susceptibility discs (Oxoid) and incubated at $37^{\circ} \mathrm{C}$ for $24 \mathrm{~h}$. The tested antibiotics were ampicillin (AMP; $10 \mu \mathrm{g}$ ), cefaclor (CEC; $30 \mu \mathrm{g}$ ), cefotaxime (CTX; $30 \mu \mathrm{g}$ ), cefoxitin (FOX; $30 \mu \mathrm{g}$ ), cefuroxime (CXM; $30 \mu \mathrm{g})$, cephazolin (KZ; $30 \mu \mathrm{g})$, ceftriaxone (CRO; $5 \mu \mathrm{g})$, ceftazidime (CAZ; $30 \mu \mathrm{g})$, cloxacillin (OB; $5 \mu \mathrm{g}$ ) imipenem (IPM; $10 \mu \mathrm{g})$, oxacillin (OX; $1 \mu \mathrm{g})$, penicillin $\mathrm{G}(\mathrm{P}$; $10 \mu \mathrm{g}$ ), and vancomycin (VA; $30 \mu \mathrm{g}$ ). The ratio of the diameters of the inhibition zones in the presence and absence of compound was used to calculate a sensitizing score for each compound/ $\beta$-lactam combination: no change in the diameter of the inhibition zones was scored as $0, a<3$-fold increase in the diameter of the inhibition zone in the presence of compound was scores as 1, while a 3-6 fold increase in the inhibition zone was scored as 2, and an increase of $>6$-fold was assigned a score of 3 . The sensitizing scores in Table 1 were obtained by adding the single scores for each compound across all $\beta$-lactams.

\section{Minimum Inhibitory Concentration}

Minimal inhibitory concentration (MIC) was determined following the Clinical and Laboratory Standards Institute 2017 guidelines in the 96-well format. Overnight cultures of $S$. aureus were diluted in physiological saline $(0.9 \% \mathrm{NaCl})$ to reach turbidity of $0.5 \mathrm{McF}$ arland (Sensititre ${ }^{\circledR}$ nephelometer and the Sensititre McFarland Standard). The bacterial suspensions were adjusted to $5 \times 10^{5} \mathrm{CFU} \mathrm{ml}^{-1}$ in cation-adjusted Mueller-Hinton broth in wells containing standard two-fold dilutions of the test compounds in a final volume of $100 \mu \mathrm{l}$. The plates were incubated for $24 \mathrm{~h}$ without shaking at $37^{\circ} \mathrm{C}$. All experiments were performed in biological triplicates. MIC was defined as the concentration of the compounds at which visible growth was completely inhibited.

\section{Checkerboard Analyses and FIC Index Determination}

FICs were determined by setting up checkerboard broth microdilution assays using TSB as the growth medium. Each compound and imipenem were serially diluted at eight different concentrations to create an $8 \times 8$ matrix. Stock solutions of BTB 00921 (5-2,500 $\left.\mathrm{mg} \mathrm{L}^{-1}\right)$, HTS 01632 (4-2000 $\left.\mathrm{mg} \mathrm{L}^{-1}\right)$, and BTB 04965 (5-2,500 $\left.\mathrm{mg} \mathrm{L}^{-1}\right)$ were prepared in DMSO. While stock solutions of imipenem $\left(50-3,200 \mathrm{mg} \mathrm{L}^{-1}\right)$ were prepared in $\mathrm{dH}_{2} \mathrm{O}$ and aliquots $(1.5 \mu \mathrm{l})$ were added to the 96 -well plate. Overnight cultures of $S$. aureus were diluted in $0.9 \% \mathrm{NaCl}$ to reach turbidity of $0.5 \mathrm{McF}$ arland (Sensititre ${ }^{\circledR}$ nephelometer and the Sensititre ${ }^{\circledR}$ McFarland Standard) and $150 \mu$ aliquots were dispensed into all
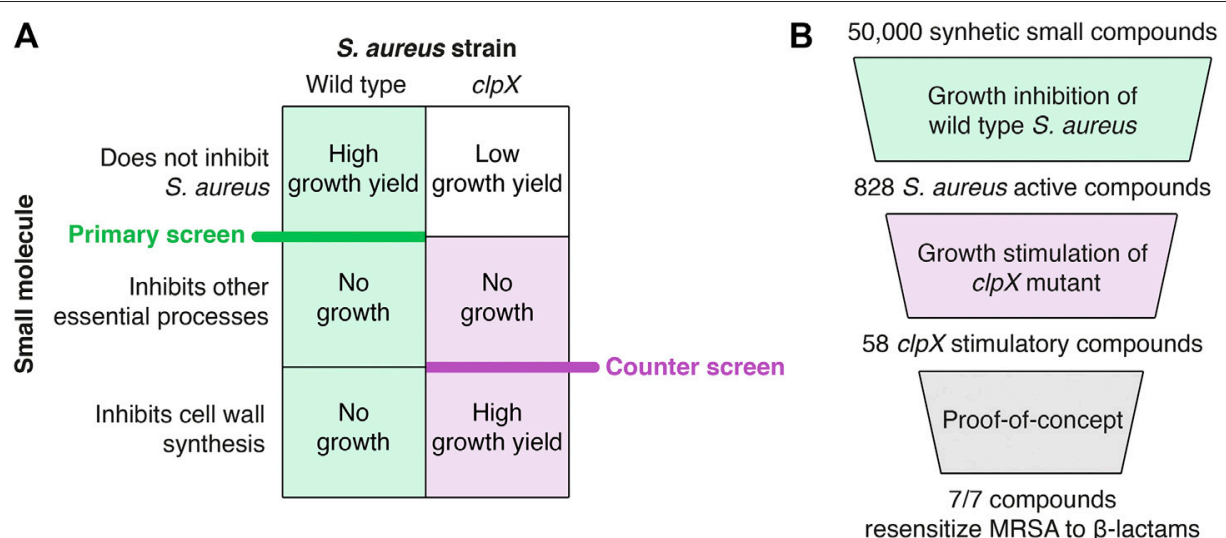

FIGURE 1 | Summary of the screening procedure. (A) Principles of the pathway-specific screen. The screen is predicted to identify inhibitors of specific steps in cell wall synthesis because such compounds inhibit the growth of the wild type strain (primary screen) while improving growth of the $c / p X$ strain (counter screen)-see text for details. (B) Screening workflow. A collection of 50,000 synthetic small molecules from the Maybridge screening collection was first screened for growth inhibition against the $S$. aureus wild type resulting in 828 active compounds. Next, a $S$. aureus clpX mutant was used in a growth-based counter-screen to identify 58 compounds capable of increasing the growth yield of the $c / p X$ mutant at $30^{\circ} \mathrm{C}$ (cut-off 1.5 fold increase in final yield as measured by optical density). Ten compounds were purchased for follow-up studies, and of these ten compounds, seven hit-compounds retained the ability to increase the final yield of $S$. aureus c/pX cultures grown at $30^{\circ} \mathrm{C}$ in a microtiter plate growth assay and sensitized the highly resistant COL MRSA to at least one $\beta$-lactam antibiotic in a disc diffusion assay (summarized in Table 1). 

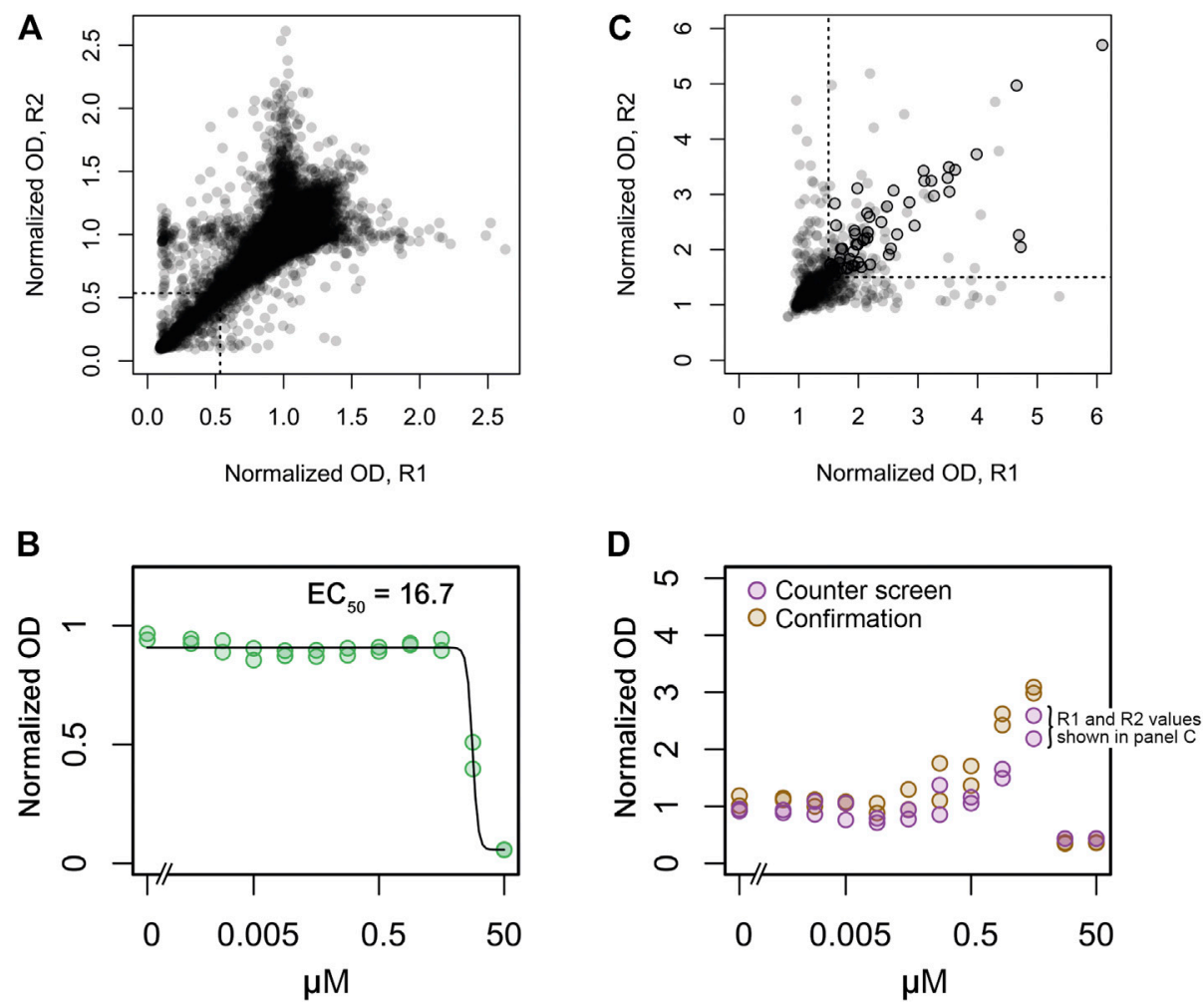

FIGURE 2 | Replicate plots and hit selection for screens of growth inhibition in wild type S. aureus and growth stimulation in the S. aureus clpX mutant. (A) A collection of 50,000 synthetic small molecules was screened at $10 \mu \mathrm{M}$ for growth inhibition of the wild type strain in duplicate. Normalized OD values for replicates 1 and 2 is depicted on the $x$ - and $y$-axes, respectively. A statistical cutoff of three standard deviations below the mean was established for both replicates, indicated by the dotted lines in the lower left corner. Data points to the left and below these lines represent the 993 active compounds. (B) 828 of the 993 active compounds were confirmed at concentrations ranging from $0.5 \mathrm{nM}$ to $50 \mu \mathrm{M}$ in duplicate. Dose-dependent inhibition by one confirmed active compound is shown as an example. Normalized OD values for the two replicates are indicated by green circles. A calculated dose-response curve (black line) and the calculated EC50 value is also shown. (C) The 828 confirmed active compounds were assessed for growth stimulation of the $c / p X$ mutant at $30^{\circ} \mathrm{C}$ at concentrations ranging from $0.5 \mathrm{nM}$ to $50 \mu \mathrm{M}$ in duplicate. For each compound the highest obtained normalized OD values of replicates 1 and 2 is depicted on the $x$ and $y$-axes, respectively, (these values are also indicated in panel $\mathrm{D}$ for one example compound). A normalized OD value of 1.5 was used as cutoff, indicated by dotted lines. Data points to the right and above these lines represent clpX stimulatory compounds, and black circles indicate the 58 compounds that were subsequently confirmed by manual inspection of dose-response plots and re-tests. (D) Dose-dependent growth stimulation by one clpX stimulatory compound (same compound as in panel B) is shown as an example.

wells. Plates were incubated at $37^{\circ} \mathrm{C}$ for $20-24 \mathrm{~h}$. The FIC for imipenem in the presence of compounds (BTB 04965, BTB 00921, or HTS 01632) was calculated in wells showing $<20 \%$ growth by dividing the concentration of imipenem in the presence of compound with the imipenem MIC in the absence of compound. The FIC index for the compound in combination with imipenem is the sum of the two FICs (White et al., 1996). FIC index $\leq 0.5$ was used to show synergism. The experiment was performed in two biological replicates.

\section{Data Analyses}

Statistical analyses were performed using R (R Core Team, 2021), and cheminformatic analyses were performed using the RDkit toolkit (https://rdkit.org) in Python 3. Pan-assay interference compounds (PAINS) were identified among the active compounds from the primary screen and the counter screen, respectively, as those compounds with a substructure matching a list of PAINS structures (https://github.com/rdkit/rdkit/blob/ master/Data/Pains/wehi_pains.csv; Saubern et al., 2011).

\section{RESULTS}

\section{Screening Concept}

The screening concept is based on the findings that the coldsensitive growth of $S$. aureus clpX mutants is rescued genetically by inactivation of LtaS, or chemically by compounds targeting TarO, catalyzing the first step in WTA synthesis, and by $\beta$-lactams binding to the trans peptidase domain of essential PBPs (Bæk et al., 2016; Jensen et al., 2019). TarO and LtaS are conditionally essential, and inactivation imposes a severe fitness cost at $37^{\circ} \mathrm{C}$ (Gründling and Schneewind, 2007; Vergara-Irigaray et al., 2008; Coe et al., 2019). We therefore reasoned that compounds targeting these crucial steps in cell wall synthesis could be identified by screening for molecules that impede growth of the wild type at $37^{\circ} \mathrm{C}$, while increasing the final growth yield of the $c l p X$ mutant at $30^{\circ} \mathrm{C}$. Hence, the screening was set up as two successive whole cell screens: a primary screen to identify compounds that inhibit growth of $S$. aureus wild type at $37^{\circ} \mathrm{C}$, and a counter screen to identify compounds that increased the final OD of an $S$. aureus $c l p X$ mutant at $30^{\circ} \mathrm{C}$ (see overview of screen in Figure 1). 


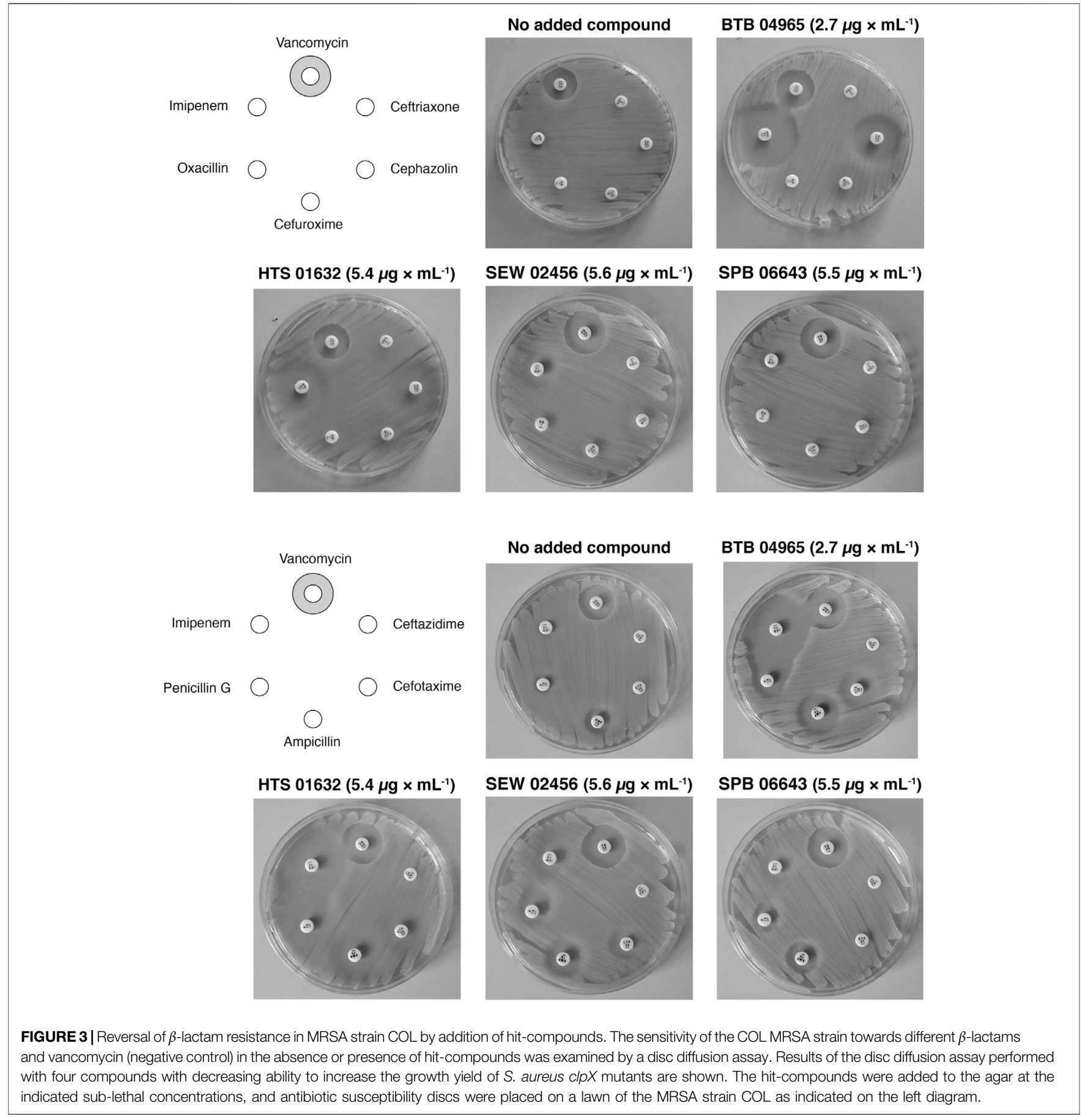

Screened out compounds targeting TarO or LTA biosynthesis are predicted to sensitize MRSA strains to $\beta$-lactams (Campbell et al., 2011; Farha et al., 2013, Roemer et al., 2013, Lee et al., 2016).

\section{Primary Screen Identifies 828 Compounds Inhibiting Growth of $S$. aureus}

The screening workflow started with a primary screen of $\sim 50,000$ small synthetic compounds from the Maybridge screening collection for growth inhibition of wild type methicillin sensitive $S$. aureus (strain SA564) at a concentration of $10 \mu \mathrm{M}$. Growth at $37^{\circ} \mathrm{C}$ was measured by change in absorbance $(600 \mathrm{~nm})$ after $8 \mathrm{~h}$ of incubation with no shaking in 384-well plates. These conditions led to an optimal screening window at late exponential growth phase (Supplementary Figure 1A). Throughout the screen, high (1\% DMSO) and low (2.5 $\mathrm{mg} \mathrm{L}^{-1}$ erythromycin) controls were included. The screening data were normalized to remove plate-to-plate and 
well-positional variation (Mangat et al., 2014). Hits were selected as those molecules causing the normalized OD values to be lower than three standard deviations below the mean of the full data set resulting in a hit rate of $2.0 \%$ and a total of 993 S. aureus active compounds (Figure 2A). Of these, 828 were confirmed as active when tested in 11 different concentrations ranging from $0.5 \mathrm{nM}$ to $50 \mu \mathrm{M}$ (Figure 2B).

\section{Counter Screen Identifies 58 Compounds That Rescue Growth of $S$. aureus clpX Mutant}

This sub-library of 828 compounds with confirmed growth-inhibitory activity against $S$. aureus was then used as a starting point in a counterscreen for growth stimulation of the $S$. aureus $\operatorname{clpX}$ mutant at $30^{\circ} \mathrm{C}$ at 11 different concentrations of each compound ranging from $0.5 \mathrm{nM}$ to $50 \mu \mathrm{M}$. Growth was measured by a change in absorbance $(600 \mathrm{~nm})$ after $24 \mathrm{~h}$ of incubation with shaking at $600 \mathrm{rpm}$ in 96 -well plates. These conditions led to an optimal screening window when we tested screening conditions with oxacillin at a concentration $\left(0.05 \mathrm{mg} \mathrm{L}^{-1}\right)$ previously shown to stimulate growth of the $c l p X$ mutant (Jensen et al., 2019; Supplementary Figure 1B). The screening data were normalized to remove plate-to-plate variation as described in Methods.

In the counter-screen, a compound was classified as active if it raised the final growth yield of the $c l p X$ mutant compared to the DMSO control by 1.5 fold or more. This cutoff-value immediately resulted in a set of 678 inactive compounds that were discarded from further analyses (Figure 2C). The doseresponse plots of the remaining compounds were then inspected manually, and the compounds were classified as either inactive, active, or inconclusive (42 compounds, Figure 2D). Fifty of the compounds were also re-tested using the same assay. In total, 58 (7\%) of the 828 compounds (or $0.12 \%$ of all screened compounds) that inhibited $S$. aureus growth also stimulated clpX growth with maximal growth yield increases ranging from 1.5 to 3.7 -fold.

\section{The Counter Screen is Efficient at Eliminating Compound Classes That Tend to Have Non-Specific Activities}

An important advantage of employing a counter screen selecting for improved growth is that nonspecific growth inhibitors are likely to be eliminated from the pool of hit compounds. A class of compounds that often show up as hits in screening campaigns, are pan-assay interference compounds (PAINS) which are chemical compounds that tend to react nonspecifically with numerous biological targets rather than specifically affecting one desired target (Baell and Holloway, 2010). Applying an in silico PAINS filter to the 58 hits shows that only two of the final hit compounds $(3 \%)$ contain a PAINS substructure, whereas this is the case for $9 \%$ of the $S$. aureus growth-inhibitory compounds that do not stimulate $\operatorname{clpX}$ growth. This result indicates that the $\operatorname{clp} X$ counter-screen is efficient at eliminating compound classes that tend to have nonspecific activities.

\section{Hit Compounds Reverse $\beta$-lactam Resistance in MRSA}

To establish a proof-of-concept, we purchased a subset of ten screening compounds and tested them for their ability to sensitize MRSA to $\beta$-lactams. The ten compounds were chosen based on their varying ability to stimulate growth of the $S$. aureus clpX strain, with the ten compounds ranking from showing no stimulation (below the 1.5 cut-off) to maximal stimulation (3.6 fold increase in final OD) in the screening set-up. We first examined if the hit compounds retained the ability to increase the growth yield of $c l p X$ cells by measuring the final OD $(600 \mathrm{~nm})$ reached by the SA564 $\operatorname{clpX}$ mutant after $24 \mathrm{~h}$ of incubation in the absence or presence of added compounds. Seven compounds met the 1.5 fold stimulation cut-off used in the secondary screen, and, in general, there was good correlation between the fold stimulation observed in this assay and the fold-stimulation determined in the original screening assay (Table 1). However, one compound (SPB 06643), which did not meet the cut-off of 1.5 fold stimulation in the original screen, showed a minor (1.6 fold) stimulation in this assay, while two compounds did not meet the 1.5 fold stimulation cut-off. We then examined the ability of the ten compounds to inhibit growth of wild type cells by determining the MICs against two different $S$. aureus wild type strains, the methicillin sensitive SA564 strain, which was used in the primary screen, and the JE2 MRSA strain belonging to the fast spreading and highly virulent community-acquired USA300 clone (Fey et al., 2013). As can be seen in Table 1, the MIC values for the compounds varied from 1 to $2 \mathrm{mg} \mathrm{L}^{-1}$ to exceeding $32 \mathrm{mg} \mathrm{L}^{-1}$ with similar MIC values measured against the JE2 MRSA strain and the methicillin sensitive SA564 strain. The high MIC values are in line with the potential inhibition of non-essential targets such as TarO. Next, we assessed if the compounds had the ability to sensitize the highly resistant MRSA strain COL to $\beta$-lactam antibiotics by doing a disc diffusion assay. In the absence of added compounds, COL displayed high resistance to all tested $\beta$-lactams antibiotics as evidenced by the absence of clearing zones surrounding the antibiotic discs (Figure 3). Remarkably, enlarged inhibition zones for one or more $\beta$-lactams was observed in the presence of sub-lethal concentrations of the seven compounds that met the 1.5 fold cut-off in the follow-up stimulation assay, demonstrating that these compounds are capable of sensitizing the MRSA strain to $\beta$-lactams (see specific examples in Figure 3, and a summary of the results in Table 1). The five most potent compounds sensitized the COL strain to four or more different types of $\beta$-lactams (Figure 4A). Notably, when we used the fold increase in the diameter of the inhibition zones in the presence and absence of compound to score the sensitizing effect for each compound/ $\beta$-lactam combination (see Methods for details and illustrated in Figure 4A) the summed sensitizing scores for each compound correlated linearly to the fold-stimulation of the SA564 clpX strain (Figure 4B). Therefore, the degree of growth stimulation of the clpX mutant seems to be a good predictor of a compound's ability to reverse $\beta$-lactam resistance. In the disc diffusion assay, the strongest sensitizing effect was observed for imipenem in combination with BTB 00921, HTS 01632, and BTB 04965 (Figure 4A and Table 1). To more directly quantify the 

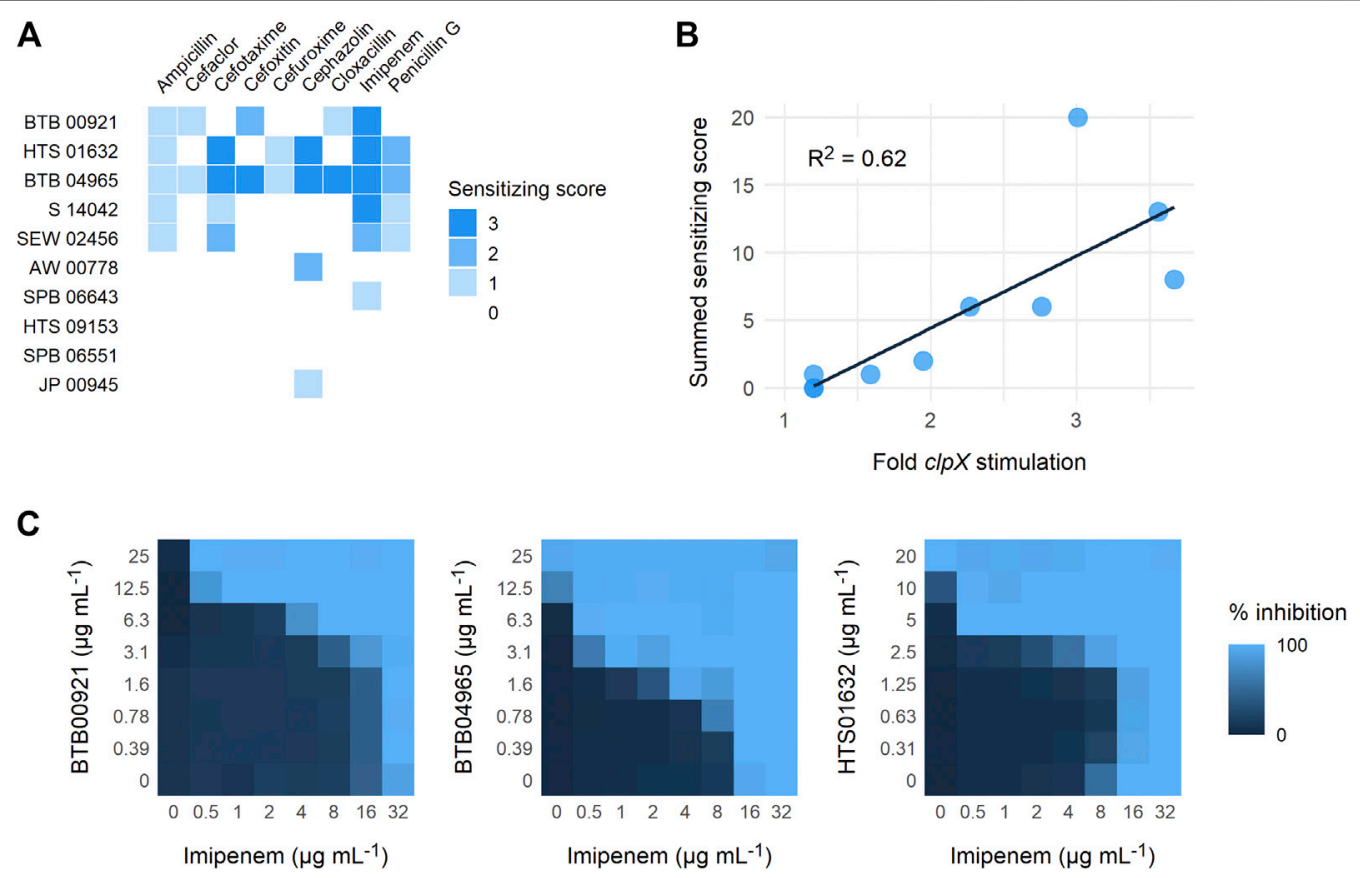

FIGURE 4 | Reversal of $\beta$-lactam resistance in MRSA strain COL. (A) The diameter of the clearing zones in the disc diffusion assay was measured and the ratio of the diameters of the inhibition zones in the presence and absence of compound were used to calculate a sensitizing score for each compound/ $\beta$-lactam combination as described in the Methods section. The compounds are listed according to their ability to stimulate growth of the clpX mutant from bottom to top. (B) The scores across all antibiotics are added to give a total synergy score for each compound. The score is plotted against the fold change in growth of $c / p X$ in the presence of compound, together with a linear regression line $\left(R^{2}=0.62\right)(\mathbf{C})$ Synergy between imipenem and the three compounds with highest sensitizing scores was evaluated by performing microdilution checkerboard analyses against the highly resistant MRSA strain, COL. The extent of inhibition is shown as a heat plot.

sensitizing effect, we finally performed checkerboard analyses for imipenem in combination with each of these three compounds (Figure 4C). We found that imipenem MIC was reduced up to 64fold in the presence of sub-inhibitory concentrations of BTB 00921, HTS 01632, or BTB 04965 (Figure 4C). Two compounds display synergy if the fractional inhibitory concentration (FIC) index, as calculated by the sum of the FIC of each compound, is $\leq 0.5$ (White et al., 1996). According to this definition, imipenem has synergy with BTB 04965 (average FIC index $=0.4$ with FIC index ranging from 0.19 to 1.0 in single wells) and with HTS 01632 (average FIC index $=0.5$ with FIC index ranging from 0.28 to 1.0 in single wells). The FIC index for BTB 00921 in combination with imipenem could not be calculated as BTB 00921 does not reach the MIC. In conclusion, our results demonstrate the efficiency of the screening setup in identifying hit-compounds that sensitize MRSA to $\beta$-lactams antibiotics.

\section{DISCUSSION}

The cell wall continues to be an excellent target for antibacterial drug discovery because of its essentiality in bacteria and its absence in mammalian cells. In this report, we describe the development and implementation of a high-throughput screening approach where a $S$. aureus mutant lacking the ClpX chaperone was used in a counter-screen to identify presumed cell wall synthesis inhibitors that at sub-inhibitory concentrations sensitize MRSA to $\beta$-lactams antibiotics. These hit-compounds could potentially be used in combination therapy with $\beta$-lactams for treatment of MRSA-infections. Additionally, some of the hit-compounds show inhibitory activity against $S$. aureus at therapeutic relevant concentration $(1-2 \mu \mathrm{M})$ and, hence, hold potential for being developed into lead compounds for mono-therapy of staphylococcal infections. However, follow up studies are needed to pin-point the precise target of the hit-compounds. Based on the findings that 1) growth of $c p X$ cells is very specifically rescued by compounds targeting TarO, PBP1 or PBP3 (Jensen et al., 2019), and that 2) spontaneous suppressor mutations only mapped in ltaS, we predicted that hit-compounds would directly or indirectly target a pathway that functionally connect TarO, PBP1/PBP3, and LtaS. So far, the molecular mechanism underlying the dramatic synergy between $\beta$-lactams and TarO inhibitors against MRSA remain unexplained. Interestingly, we here observed very good correlation between the ability of the hit-compounds to increase the growth yield of the $c l p X$ mutant, and the ability of the compounds to sensitize MRSA to $\beta$-lactam antibiotics. The sensitizing effect varied widely between different types of $\beta$-lactams as was previously shown for TarO inhibitors (Campbell et al., 2011; Farha et al., 2013). For all compounds, the strongest sensitizing effect was observed with imipenem that is specific for $S$. aureus PBP1 whose function is confined to synthesis of the septal wall (Reichmann et al., 2019). Strikingly, imipenem is also superior to other $\beta$-lactams in improving growth of clpX cells (Jensen et al., 2019). Taken 
together, these correlations point to a functional connection between the early steps of WTA biosynthesis and the transpeptidase domain of PBPs that is critical for both the synergy between TarO inhibitors and $\beta$-lactams, and for alleviating the cold-sensitive growth defect of $\operatorname{clpX}$ cells. Inactivation of $\operatorname{clp} X$ results in accumulation of the Sle1 cell wall hydrolase involved in separation of $S$. aureus daughter cells (Thalsø-Madsen et al., 2019). The severe growth defect of clpX cells was explained by showing that at $30^{\circ} \mathrm{C}$, a combination of aberrant septum synthesis and high Sle1 levels caused premature splitting of daughter cells resulting in cell lysis (Jensen et al., 2019). Remarkably, $\beta$-lactams prevented Sle1 dependent lysis of clpX cells (Jensen et al., 2019). As also WTA and LTA have a crucial role in promoting septal localization of autolysins, the ability to antagonize Sle1 mediated lysis could be a central feature in providing $c l p X$ stimulation (Schlag et al., 2010; Zoll et al., 2012). Therefore, our $c l p X$ based counter screen may select broadly for compounds that impede autolytic splitting of daughter cells. The mechanisms coordinating cell wall hydrolase activity with peptidoglycan synthesis are crucial for bacterial viability, however, relatively little is known about the check points that safeguard bacteria from the detrimental activity of cell wall hydrolases during the cell cycle. We hope that a further characterization of the hit-compounds identified in this study will bring novel insight into these important mechanisms.

\section{REFERENCES}

Baell, J. B., and Holloway, G. A. (2010). New Substructure Filters for Removal of Pan Assay Interference Compounds (PAINS) from Screening Libraries and for Their Exclusion in Bioassays. J. Med. Chem. 53, 2719-2740. doi:10.1021/ jm901137j

Bæk, K. T., Bowman, L., Millership, C., Dupont Søgaard, M., Kaever, V., Siljamäki, P., et al. (2016). The Cell Wall Polymer Lipoteichoic Acid Becomes Nonessential in Staphylococcus aureus Cells Lacking the ClpX Chaperone. mBio. 7, e01228-16. doi:10.1128/mBio.01228-16

Baker, T. A., and Sauer, R. T. (2012). ClpXP, an ATP-Powered Unfolding and Protein-Degradation Machine. Biochim. Biophys. Acta (Bba) - Mol. Cel Res. 1823, 15-28. doi:10.1016/j.bbamcr.2011.06.007

Bickerton, G. R., Paolini, G. V., Besnard, J., Muresan, S., and Hopkins, A. L. (2012). Quantifying the Chemical beauty of Drugs. Nat. Chem. 4, 90-98. doi:10.1038/ nchem. 1243

Buss, J. A., Baidin, V., Welsh, M. A., Flores-Kim, J., Cho, H., Wood, B. M., et al. (2018). Pathway-Directed Screen for Inhibitors of the Bacterial Cell Elongation Machinery. Antimicrob. Agents Chemother. 63, e01530-18. doi:10.1128/AAC. 01530-18

Campbell, J., Singh, A. K., Santa Maria, J. P., Kim, Y., Brown, S., Swoboda, J. G., et al. (2011). Synthetic Lethal Compound Combinations Reveal a Fundamental Connection between Wall Teichoic Acid and Peptidoglycan Biosyntheses inStaphylococcus Aureus. ACS Chem. Biol. 6, 106-116. doi:10. $1021 / \mathrm{cb} 100269 \mathrm{f}$

Coe, K. A., Lee, W., Stone, M. C., Komazin-Meredith, G., Meredith, T. C., Grad, Y. H., et al. (2019). Multi-strain Tn-Seq Reveals Common Daptomycin Resistance Determinants in Staphylococcus aureus. Plos Pathog. 15, e1007862, doi:10.1371/ journal.ppat.1007862

Dyke, K. G. H., Jevons, M. P., and Parker, M. T. (1966). Penicillinase Production and Intrinsic Resistance to Penicillins in Staphylococcus aureus. The Lancet. 287, 835-838. doi:10.1016/s0140-6736(66)90182-6

Farha, M. A., Leung, A., Sewell, E. W., D’Elia, M. A., Allison, S. E., Ejim, L., et al. (2013). Inhibition of WTA Synthesis Blocks the Cooperative Action of PBPs

\section{DATA AVAILABILITY STATEMENT}

The original contributions presented in the study are included in the article/Supplementary Material, further inquiries can be directed to the corresponding author.

\section{AUTHOR CONTRIBUTIONS}

$\mathrm{DF}$ and $\mathrm{KB}$ wrote the manuscript. $\mathrm{DF}, \mathrm{KB}, \mathrm{MF}$, and $\mathrm{EB}$ designed the screen, and interpreted the results. $\mathrm{DF}$ and $\mathrm{CJ}$ supervised the students. KB and MF performed the screen. MV, CJ, VM, TB, and $\mathrm{EP}$ performed the follow up experiments.

\section{FUNDING}

This work was funded by grants from The Danish Research Council (DFF, FTP), grants 0136-00200B, 4184-00033 and 12132527 to DF and KB.

\section{SUPPLEMENTARY MATERIAL}

The Supplementary Material for this article can be found online at: https://www.frontiersin.org/articles/10.3389/fmolb.2021.691569/ full\#supplementary-material

and Sensitizes MRSA to $\beta$-Lactams. ACS Chem. Biol. 8, 226-233. doi:10.1021/ cb300413m

Fey, P. D., Endres, J. L., Yajjala, V. K., Widhelm, T. J., Boissy, R. J., Bose, J. L., et al. (2013). A Genetic Resource for Rapid and Comprehensive Phenotype Screening of Nonessential Staphylococcus aureus Genes. mBio. 4, e00537-12. doi:10.1128/ mBio.00537-12

Frees, D., Qazi, S. N. A., Hill, P. J., and Ingmer, H. (2003). Alternative Roles of ClpX and ClpP in Staphylococcus aureus Stress Tolerance and Virulence. Mol. Microbiol. 48, 1565-1578. doi:10.1046/j.1365-2958.2003.03524.x

French, S., Ellis, M. J., Coutts, B. E., and Brown, E. D. (2017). Chemical Genomics Reveals Mechanistic Hypotheses for Uncharacterized Bioactive Molecules in Bacteria. Curr. Opin. Microbiol. 39, 42-47. doi:10.1016/j.mib.2017.09.005

Gründling, A., and Schneewind, O. (2007). Synthesis of Glycerol Phosphate Lipoteichoic Acid in Staphylococcus aureus. Proc. Natl. Acad. Sci. 104, 8478-8483. doi:10.1073/pnas.0701821104

Jelsbak, L., Ingmer, H., Valihrach, L., Cohn, M. T., Christiansen, M. H. G., Kallipolitis, B. H., et al. (2010). The Chaperone ClpX Stimulates Expression of Staphylococcus aureus Protein A by Rot Dependent and Independent Pathways. PLoS One 5, e12752. doi:10.1371/journal.pone.0012752

Jensen, C., Bæk, K. T., Gallay, C., Thalsø-Madsen, I., Xu, L., Jousselin, A., et al. (2019). The ClpX Chaperone Controls Autolytic Splitting of Staphylococcus aureus Daughter Cells, but Is Bypassed by $\beta$-lactam Antibiotics or Inhibitors of WTA Biosynthesis. Plos Pathog. 15, e1008044. doi:10.1371/journal.ppat. 1008044

Lee, S. H., Wang, H., Labroli, M., Koseoglu, S., Zuck, P., Mayhood, T., et al. (2016). TarO-specific Inhibitors of wall Teichoic Acid Biosynthesis Restore $\beta$-lactam Efficacy against Methicillin-Resistant Staphylococci. Sci. Transl. Med. 8, 329ra32. doi:10.1126/scitranslmed.aad7364

Mangat, C. S., Bharat, A., Gehrke, S. S., and Brown, E. D. (2014t). Rank Ordering Plate Data Facilitates Data Visualization and Normalization in HighThroughput Screening. J. Biomol. Screen. 19 (9), 1314-1320. doi:10.1177/ 1087057114534298

Olivares, A. O., Baker, T. A., and Sauer, R. T. (2016). Mechanistic Insights into Bacterial AAA+ Proteases and Protein-Remodelling Machines. Nat. Rev. Microbiol. 14, 33-44. doi:10.1038/nrmicro.2015.4 
R Core Team (2021). R: A Language and Environment for Statistical Computing. Vienna, Austria: R Foundation for Statistical Computing. Available at: https:// www.R-project.org/

Reichmann, N. T., Tavares, A. C., Saraiva, B. M., Jousselin, A., Reed, P., Pereira, A. R., et al. (2019). SEDS-bPBP Pairs Direct Lateral and Septal Peptidoglycan Synthesis in Staphylococcus aureus. Nat. Microbiol. 4, 1368-1377. doi:10.1038/ s41564-019-0437-2

Richter, S. G., Elli, D., Kim, H. K., Hendrickx, A. P. A., Sorg, J. A., Schneewind, O., et al. (2013). Small Molecule Inhibitor of Lipoteichoic Acid Synthesis Is an Antibiotic for Gram-Positive Bacteria. Proc. Natl. Acad. Sci. USA. 110, 3531-3536. doi:10.1073/pnas.1217337110

Roemer, T., Schneider, T., and Pinho, M. G. (2013). Auxiliary Factors: a Chink in the Armor of MRSA Resistance to $\beta$-lactam Antibiotics. Curr. Opin. Microbiol. 16, 538-548. doi:10.1016/j.mib.2013.06.012

Saubern, S., Guha, R., and Baell, J. B. (2011). KNIME Workflow to Assess PAINS Filters in SMARTS Format. Comparison of RDKit and Indigo Cheminformatics Libraries. Mol. Inf. 30, 847-850. doi:10.1002/minf.201100076

Schlag, M., Biswas, R., Krismer, B., Kohler, T., Zoll, S., Yu, W., et al. (2010). Role of Staphylococcal wall Teichoic Acid in Targeting the Major Autolysin Atl. Mol. Microbiol. 75, 864-873. doi:10.1111/j.1365-2958.2009.07007.x

Sewell, E. W., and Brown, E. D. (2014). Taking Aim at wall Teichoic Acid Synthesis: New Biology and New Leads for Antibiotics. J. Antibiot. 67, 43-51. doi:10.1038/ja.2013.100

Silver, L. L. (2011). Challenges of Antibacterial Discovery. Clin. Microbiol. Rev. 24, 71-109. doi:10.1128/CMR.00030-10

Somerville, G. A., Beres, S. B., Fitzgerald, J. R., DeLeo, F. R., Cole, R. L., Hoff, J. S., et al. (2002). In Vitro serial Passage of Staphylococcus aureus: Changes in Physiology, Virulence Factor Production, and Agr Nucleotide Sequence. J Bacteriol. 184, 1430-1437. doi:10.1128/JB.184.5.1430-1437.2002

Tacconelli, E., Carrara, E., Savoldi, A., Harbarth, S., Mendelson, M., Monnet, D. L., et al. (2018). Discovery, Research, and Development of New Antibiotics: the WHO Priority List of Antibiotic-Resistant Bacteria and Tuberculosis. Lancet Infect. Dis. 18, 318-327. doi:10.1016/S1473-3099(17)30753-3
Thalsø-Madsen, I., Torrubia, F. R., Xu, L., Petersen,, A., Jensen, C., and Frees, D (2019). The Sle1 Cell Wall Amidase Is Essential for $\beta$-Lactam Resistance in Community-Acquired Methicillin-Resistant Staphylococcusaureus USA300. Antimicrob. Agents Chemother. 64, e01931-19. doi:10.1128/AAC.01931-19

Vergara-Irigaray, M., Maira-Litrán, T., Merino, N., Pier, G. B., Penadés, J. R., and Lasa, I. (2008). Wall Teichoic Acids Are Dispensable for Anchoring the PNAG Exopolysaccharide to the Staphylococcus aureus Cell Surface. Microbiology. 154, 865-877. doi:10.1099/mic.0.2007/013292-0

Vestergaard, M., Frees, D., and Ingmer, H. (2019). Antibiotic Resistance and the MRSA Problem. Microbiol. Spectr. 7. doi:10.1128/microbiolspec.GPP3-00572018

White, R. L., Burgess, D. S., Manduru, M., and Bosso, J. A. (1996). Comparison of Three Different In Vitro Methods of Detecting Synergy: Time-Kill, Checkerboard, and E Test. Antimicrob. Agents Chemother. 40, 1914-1918. doi:10.1128/aac.40.8.1914

Zoll, S., Schlag, M., Shkumatov, A. V., Rautenberg, M., Svergun, D. I., Götz, F., et al. (2012). Ligand-binding Properties and Conformational Dynamics of Autolysin Repeat Domains in Staphylococcal Cell wall Recognition. J. Bacteriol. 194, 3789-3802. doi:10.1128/JB.00331-12

Conflict of Interest: The authors declare that the research was conducted in the absence of any commercial or financial relationships that could be construed as a potential conflict of interest.

Copyright (c) 2021 Bak, Jensen, Farha, Nielsen, Paknejadi, Mebus, Vestergaard, Brown and Frees. This is an open-access article distributed under the terms of the Creative Commons Attribution License (CC BY). The use, distribution or reproduction in other forums is permitted, provided the original author(s) and the copyright owner(s) are credited and that the original publication in this journal is cited, in accordance with accepted academic practice. No use, distribution or reproduction is permitted which does not comply with these terms. 